\title{
La pathologie tissulaire de John Hunter
}

\author{
Par Othmar Keel
}

Depuis le $\mathrm{XIX}^{\mathrm{e}}$ siècle, on a toujours, en histoire de la médecine, décrit la naissance de la pathologie tissulaire dans des termes analogues aux suivants:

... la décomposition des divers appareils en membranes distinctes et en tissus particuliers, et la constitution de groupes de phlegmasies fondés sur la différence de ces tissus sont des idées géniales. Pinel, sur ce point fut le précurseur de Bichat. Celui-ci, avec une admirable sagacité, s'empara, en effet, de cette idée et fonda l'anatomie générale. Ici, le clinicien avait devancé le physiologiste ${ }^{1}$.

Est-il bien vrai que ces idées géniales sont de Philippe Pinel (1745-1826) et que personne avant lui ne les avait émises? On sait que c'est Xavier Bichat (17711802) lui-même qui a attribué à Pinel l'idée fondatrice de la pathologie tissulaire. On cite toujours à ce propos la phrase suivante de l'auteur du Traité des Membranes (1800):

C'est en lisant son ouvrage [il s'agit de la Nosographie philosophique (1798) de Pinel] que l'idée de celui-ci s'est présentée à moi ${ }^{2}$.

Mais l'on n'a jamais prêté attention, semble-t-il, à ce que Bichat avait écrit juste avant cette phrase. Or, il y a là un passage qui nous semble de la plus grande importance. Bichat commence par reconnaître que Haller avait déjà distingué, en anatomie, les membranes des organes qu'elles constituent. Cependant, observet-il, «il [Haller] n'établit dans son article sur les membranes en général aucune ligne de démarcation entre elles. Une texture analogue les confond toutes, ... ${ }^{3}$ Ce à quoi il objecte qu'il y a entre les membranes différence de composition comme de fonction:

Cette composition pourrait-elle être en effet la même dans des parties que distinguent leur conformation extérieure, leurs propriétés vitales, leurs fonctions ? ${ }^{4}$

Et l'auteur du Traité des membranes nous apprend que cette vérité de l'anatomie et de la physiologie a été reconnue depuis Haller par plusieurs cliniciens très connus:

Plusieurs médecins célèbres ont conçu cette vérité depuis Haller; ils ont senti que, dans le système membraneux, diverses limites étaient à établir entre des organes jusqu'ici confondus. L'observation des caractères extrêmement variés que prend l'inflammation sur chaque membrane leur en a surtout indiqué la nécessité; car souvent,l'état morbifique plus que l'état sain, développe nettement la différence des organes entre eux, parce que dans l'un, plus que dans l'autre cas, leurs forces vitales se montrent très prononcées. 
M. Pinel a établi d'après ces principes un judicieux rapprochement entre la structure différente et les différentes affections des membranes: c'est en lisant son ouvrage..., etc. ${ }^{5}$

Il y a lieu donc de se demander pourquoi les historiens de la médecine ont toujours retenu la fin de ce passage et jamais le début. En effet, les premières lignes de ce texte établissent, de manière indiscutable, qu'un certain nombre de médecins avaient observé avant Pinel que l'inflammation diffère selon les membranes qu'elle affecte et que c'est d'après ces principes judicieux que l'auteur de la Nosographie philosophique a pu fonder la distinction des maladies selon les tissus divers de l'économie animale.

On peut noter qu'il est encore un autre passage où Bichat fait référence à l'expérience des cliniciens avant Pinel, pour étayer le principe que même dans les cas où l'on ne prend en considération qu'un seul et même système de tissus (ici le système muqueux) il faut admettre qu'il y a des affections qui n'en affectent que certaines parties:

En rapportant ainsi à deux membranes générales toutes les surfaces muqueuses, je suis non seulement appuyé sur l'inspection anatomique, mais encore l'observation pathologique me fournit et des points de démarcation entre elles deux, et des points de contact entre les diverses portions de membranes dont chacune est l'assemblage. Dans les divers tableaux d'épidémies catarrhales traçés par les auteurs, on voit fréquemment l'une de ces membranes être affectée en totalité, l'autre au contraire rester intacte; il n'est surtout pas rare d'observer une affection générale de la première, de celle qui se prolonge de la bouche, du nez et de la surface de l'œil dans les voies alimentaires et les bronches. La dernière épidémie observée à Paris, dont M. Pinel a été lui-même affecté, portait ce caractère; celle de 1761, décrite par Razous, le présentait aussi celle de 1732, décrite dans les Mémoires de la Société d'Edimbourg, fut remarquable par un semblable phénomène: or, on ne voit point alors une affection correspondante dans la membrane muqueuse qui se déploie dans les organes urinaires et sur ceux de la génération. Il y a done ici 1) analogie entre les portions de la première par l'uniformité d'affection; 2) démarcation entre les deux par l'intégrité de l'une et la maladie de l'autre ${ }^{6}$.

Ce qui signifie que Bichat savait parfaitement qu'avant Pinel et lui-même, un certain nombre de médecins s'étaient déjà intéressés à la pathologie tissulaire - soit aux membranes et à leurs altérations - et qu'ils avaient déjà conçu l'idée que le siège de la maladie se situe plutôt au niveau des différents tissus qui composent l'organe qu'au niveau de l'organe en son entier.

Ce qui est tout à fait symptomatique, c'est que le début du texte dans lequel Bichat reconnaît sa dette à l'égard de Pinel a été systématiquement occulté non seulement par les historiens de la médecine, mais encore par les contemporains de l'auteur du Traité des Membranes. Ainsi, au début du XIX ${ }^{\mathrm{e}}$ siècle, G. Cuvier écrit-il: 
Bichat avait été conduit à sa méthode par les leçons de Pinel, qui avait essayé de fonder la pathologie sur la distinction des différents tissus, et sur les affections dont ces tissus sont le siège ${ }^{7}$.

Mais Cuvier déjà omet de nous faire savoir ce que nous avait appris Bichat, à savoir que Pinel a été conduit lui-même à cette problématique par les observations judicieuses de plusieurs médecins qui l'avaient précédé. Il est donc légitime, semble-t-il, de chercher à savoir qui ont pu être ces médecins qui ont élaboré les premiers principes de la pathologie tissulaire.

Dans un essai récent ${ }^{8}$, nous avons montré que les idées de la décomposition des divers organes en tissus distincts et la constitution de groupes de maladies fondés sur la différence de ces tissus n'appartenaient en fait pas à Pinel mais à un médecin écossais auquel l'auteur de la Nosographie philosophique les a «empruntées». Ce médecin écossais, que Pinel n'a jamais cité, est James Carmichael Smyth (17461821) auteur d'un Mémoire intitulé Of the Different Kinds or Species of Inflammation, and of the Causes to Which they may be ascribed (Medical Communications, vol.2, Londres, 1790). Smyth est un des médecins qui, avant Pinel, en observant les caractères extrêmement variés que prend l'inflammation sur chaque membrane ont compris que «dans le système membraneux, diverses limites étaient à établir entre des organes jusqu'ici confondus». ${ }^{9}$ Mais Bichat ignorait «l'emprunt» de Pinel à Smith. Ce dernier n'était pas célèbre en France. C'est donc à d'autres médecins que Bichat fait référence. Il reste donc légitime et nécessaire de chercher de qui il s'agit. Si Bichat ignorait l'œuvre de Smyth, il connaissait par contre les travaux des élèves de l'école anatomique fondée par Hunter à Londres vers le milieu du XVIII ${ }^{\mathrm{e}}$ siècle. Parmi les élèves de William Hunter (1718-1783), le plus célèbre était sans aucun doute son propre frère: John Hunter (1728-1793) ${ }^{10}$. Or, nous allons voir que chez ce dernier auteur, on trouve déjà (comme chez J.C. Smyth qui s'est d'ailleurs inspiré en partie de Hunter ${ }^{11}$ ) une problématique tissulaire en pathologie. De cette problématique, nous allons exposer maintenant les grandes lignes.

Dans ses Leçons sur les Principes de la chirurgie ${ }^{12}$, ouvrage tiré d'un cours professé à partir de 1772 et dont l'édition a été établie sur la base d'une sténographie de ce cours recueillie en 1786 et $1787^{13}$, Hunter distingue l'inflammation en simple et composée. L'inflammation est dite simple quand «il n'y a qu'un seul mode d'action dans la partie enflammée »; ${ }^{14}$ elle est dite composée «quand il y a deux modes d'action ou un plus grand nombre ${ }^{15}$ L'inflammation composée produit trois grands effets dans les tissus: l'adhérence, la suppuration et l'ulcération. Dès 
le début du chapitre intitulé «De l'inflammation en général», l'auteur nous apprend qu'il se propose d'analyser l'inflammation adhésive selon les caractères qu'elle prend dans les différents tissus:

... j'étudierai successivement cette inflammation 1) dans le tissu cellulaire en général et dans les cavités sans ouvertures; 2) dans les conduits qui font communiquer l'intérieur du corps avec l'extérieur comme la bouche, le nez, la conjonctive, etc., et dans les canaux excréteurs des glandes, en un mot dans les tissus qui ont été appelés membranes muqueuses $^{16}$.

D’une manière plus générale, Hunter établit dès les premières lignes de ce chapitre que le processus de l'inflammation dans les membranes séreuses et dans le tissu cellulaire produit presque toujours en premier l'effet de l'adhérence, qui peut être suivi par la suppuration et l'ulcération, tandis que dans les membranes muqueuses, l'inflammation produit d'abord l'effet de la suppuration, qui n'est suivi de l'effet de l'adhérence que si l'inflammation prend une très grande ampleur:

Quant l'inflammation a son siège dans le premier ordre de tissus [il s'agit du tissu cellulaire et des membranes séreuses que Hunter appelle tour à tour cavités circonscrites, cavités séreuses, surfaces séreuses, etc.] elle se présente communément sous la forme de l'inflammation adhésive, mais c'est l'inflammation suppurative ou l'inflammation ulcérative qui succède à cette dernière suivant les circonstances et probablement suivant l'intensité du travail phlegmasique.

Dans le second ordre de tissus [il s'agit des membranes muqueuses], le travail de suppuration s'établit le premier et il ne se manifeste point de travail adhésif, à moins que l'inflammation ne soit portée très haut ${ }^{17}$.

Dans le tissu cutané, le processus de l'inflammation diffère et de la manière dont il s'opère dans le tissu cellulaire et les séreuses et de celle dont il s'opère dans les muqueuses: ici, c'est l'ulcération qui est l'effet principal:

Quant l'inflammation se montre avec violence à la surface de la peau; le travail ulcératif précède le travail de suppuration: c'est précisément le contraire de ce qui se passe dans le tissu cellulaire ${ }^{18}$.

Il y a encore d'autres effets distincts de l'inflammation selon qu'elle a son siège dans les membranes séreuses ou dans les membranes muqueuses. Ainsi dans les séreuses, si l'inflammation ne s'arrête pas à la période adhésive - ce qui constitue normalement sa résolution naturelle - elle passe alors au stade de la suppuration. La sécrétion du pus, dans les membranes séreuses, constitue la première phase de «la formation d'une nouvelle substance qu'on appelle granulations tandis que la suppuration ne produit pas nécessairement de granulations dans «les canaux tapissés par une membrane muqueuse $» .^{19}$ 
L'auteur des Principes de la chirurgie a judicieusement observé les différences qui existent entre divers autres tissus dans le processus inflammatoire dont ils sont le siège. Ainsi, dans le tissu veineux, l'inflammation peut ne pas dépasser la période adhésive; quant à la suppuration elle peut "présenter tous les degrés de violence et d'extension » ou être très limitée. Dans le tissu artériel, par contre, il semble qu'il n'y ait jamais de suppuration ${ }^{20}$.

Pour ce qui est du tissu osseux, si l'inflammation peut s'y manifester selon les différents effets de l'adhérence, de la suppuration et de l'ulcération, l'inflammation adhésive se spécifie dans ce tissu par un phénomène particulier: la formation de matière osseuse nouvelle dans les parties enflammées ${ }^{21}$. C'est pourquoi, dit l'auteur, on peut qualifier d'inflammation ossifique celle qui a son siège dans ce tissu. Il y a aussi des symptômes propres à l'inflammation du tissu osseux: la douleur qu'elle produit est plus violente que dans les autres parties ${ }^{22}$. Autre particularité de l'inflammation dans le tissu osseux: la suppuration peut s'y établir, mais beaucoup moins facilement que dans les autres tissus. Ainsi, Hunter observe que la suppuration se produit plus facilement dans le périoste (qui appartient au système du tissu fibreux) que dans l'os lui-même ${ }^{23}$.

Pour ce qui est des articulations, Hunter observe que ce sont des cavités circonscrites, et qu'appartenant donc au système séreux, elles sont sujettes au même mode de lésion et de maladie que les autres membranes séreuses. Cependant, «leur structure particulière amène quelques fois des différences dans les accidents consécutifs. ${ }^{24} \mathrm{~A}$ la différence des autres membranes séreuses, les articulations ne contractent que difficilement l'inflammation adhésive «parce que cette inflammation aurait pour conséquence la perte du mouvement dans une partie qui a été primitivement destinée au mouvement $»{ }^{25}$ Par ailleurs, les articulations en raison de leur composition guérissent plus lentement que les autres séreuses et «elles sont sujettes à des maladies spécifiques qu'elles doivent aux tissus qui entrent dans leur structure et dont la guérison est difficile à obtenir.» ${ }^{26}$ Les inflammations des articulations ont les caractéristiques suivantes qui les distinguent de celles des autres organes:

Dans toutes les inflammations articulaires, il y a du gonflement à cause de l'extravasation qui se fait dans le tissu cellulaire et dans la cavité de l'articulation, comme on le voit au genou; au-dessus de la rotule, où il y a une bourse muqueuse ${ }^{27}$.

L'inflammation est beaucoup plus dangereuse dans les articulations que dans les autres membranes séreuses, car «n'étant point limitée par le travail adhésif, elle doit ainsi que la suppuration qu'elle amène à sa suite, s'étendre à toute la cavité».28 
Hunter a souligné aussi que toutes les maladies évoluaient de manière spécifique dans les tissus de nouvelle formation. En effet, «ces tissus se détruisent plus facilement que les tissus naturels, parce que les actions vitales y sont moins énergiques. ${ }^{29}$ En conséquence, «dans ces tissus, les maladies parcourent leurs périodes beaucoup plus rapidement que dans les parties primitives.» ${ }^{30}$ Notre auteur observe que «les tissus accidentels» sont plus disposés que les autres à se gangrener et à suppurer, mais que ces altérations restent limitées à ces tissus : «la gangrène reste bornée au tissu accidentel, excepté dans les constitutions très détériorées. ${ }^{31}$

A l'actif encore de Hunter, il faut reconnaître qu'il a établi avec une grande précision anatomo-clinique, les différences du processus morbide de l'ulcération selon les différents tissus:

Certaines parties de nos solides ont plus de susceptibilité que les autres pour être absorbées, surtout par voie d'ulcération... tels sont en particulier le tissu cellulaire et le tissu adipeux, car on trouve souvent les muscles, les tendons et les ligaments dépouillés par l'ulcération du tissu cellulaire qui leur sert de moyen d'union, comme on peut l'observer quand on ouvre un vaste abcès. La peau elle-même, lorsqu'elle est soumise à une compression extérieure, est moins facilement absorbée que le tissu cellulaire... ${ }^{32}$

Dans les Leçons sur les principes de chirurgie et dans les autres ouvrages de Hunter, il y a une transcription très fréquente d'observations d'histologie pathologique aussi fines et précises (pour l'époque) que la suivante:

Je crois qu'il ne peut se former de granulations à la surface interne d'aucun conduit muqueux comme conséquence de la suppuration, à moins qu'il n'y ait une solution de continuité, et ici, je puis faire observer que quand le développement des granulations se fait à la surface d'un tel canal, ce n'est pas la membrane propre du canal, mais le tissu cellulaire sous-muqueux qui les produit ${ }^{33}$.

C'est d'ailleurs en comparant les modes différentiels du processus morbide dans les différents tissus que Hunter parvient à définir les caractères anatomopathologiques de l'inflammation:

Il résulte de ce qui se passe dans la suppuration des membranes séreuses et des surfaces muqueuses qu'une perte de substance n'est point une condition nécessaire pour la suppuration ${ }^{34}$.

C'est donc avec étonnement que l'on lira maintenant à l'article «Membrane» (1819) du Dictionnaire des sciences médicales ${ }^{35}$ que:

Les phlegmasies des membranes muqueuses ont entre elles des traits d'analogies extrêmement remarquables; l'idée de les grouper en un même ordre est un trait de génie de M. le Professeur Pinel ${ }^{36}$. 
En effet, longtemps avant Pinel (et Bichat), Hunter et Smyth avaient montré qu'il y a la plus grande analogie du mode et de la marche de l'inflammation dans les différentes membranes muqueuses (Cf. Keel, op. cit., chap. IV et V). Dans son Traité du sang, de l'inflammation et des plaies par armes à feu (1794) ${ }^{37}$, publié un an après sa mort, mais rédigé pour la première fois en $1762^{38}$, Hunter a décrit les traits caractéristiques du processus inflammatoire dans le système muqueux:

Dans les canaux internes, où les adhérences seraient nuisibles le plus souvent, les parties contractent immédiatement l'inflammation suppurative, et l'inflammation adhésive en est communément exclue. Les parties de cette espèce sont: la surface interne des paupières, du nez, de la bouche, de la trachée, des cellules aériennes des poumons, de l'œsophage, de l'estomac, des intestins, du bassinet des reins, des uretères, de la vessie de l'urètre, de l'utérus, du vagin: en un mot de tous les conduits et de tous les canaux excréteurs des organes de sécrétion, parmi lesquels toutes les parties ci-dessus mentionnées peuvent être classées jusqu'à un certain point, et qui sont désignées communément par le nom de membranes muqueuses. Dans les parties de cette espèce, si l'inflammation est légère, c'est l'inflammation suppurative qui se développe, et elle prend naissance presque immédiatement, car elle n'est pas retardée par la période adhésive; c'est ce qui rend compte de la rapidité avec laquelle la suppuration survient fréquemment dans ces parties $^{39}$.

On le voit, les membranes muqueuses forment chez Hunter un système histologique homogène, dans la mesure où il y a analogie du mode d'inflammation dans les différents éléments de ce système, quelle que soit la région du corps où ils se trouvent. Ce principe s'applique aussi aux éléments du tissu séreux. En 1801, dans l'Anatomie générale appliquée à la physiologie et à la médecine, Bichat écrira à ce propos:

Que le tissu séreux appartienne au cerveau par l'arachnoïde, au poumon par la plèvre, au cœur par le péricarde, aux viscères gastriques par le péritoine etc., cela est indifférent: partout il s'enflamme de la même manière... Pinel me paraît avoir beaucoup fait pour l'art, en commençant le premier à présenter les inflammations par ordre de systèmes, et en embrassant d'un coup d'œil général toutes celles du même système quelle que soient les organes où celui-ci se trouve ${ }^{40}$.

Or, avant Pinel, Hunter avait montré que l'inflammation a la même structure dans toutes les membranes séreuses (Hunter désigne les séreuses du nom de cavités circonscrites ou parfois de surfaces séreuses):

Toutes les cavités circonscrites, comme la plèvre, le péritoine, etc., sont susceptibles des trois inflammations. Il peut s'y former des abcès véritables et distincts, dont la matière est renfermée par des adhérences, comme dans les abcès ordinaires. Si l'inflammation ne s'arrête pas à la période adhésive, la suppuration se forme, et l'ulcération devient également nécessaire pour la sortie du pus, qui est une substance étrangère. Un fait de 
haute importance dans l'inflammation des cavités qui contiennent des parties vitales, c'est qu'il y a très peu de sympathie entre les parties enveloppantes et les parties enveloppées, d'où il résulte que l'inflammation de la première ne se propage pas toujours aux autres: ainsi l'inflammation du feuillet pariétal et du feuillet viscéral du péritoine peut exister sans l'inflammation de la tunique interne de l'intestin. Mais ce fait n'est pas universellement vrai car l'inflammation de la tunique vaginale s'étend à toute l'épaisseur du corps du testicule ${ }^{41}$.

Après avoir mis en évidence l'identité des traits de l'inflammation sur les membranes séreuses, Hunter examine tour à tour plus en détail le processus inflammatoire dont chacune est le siège. C'est ainsi qu'il examine la plèvre, le péricarde, le péritoine etc. ${ }^{42}$ : ce qui montre bien qu'elles forment pour notre auteur un système sous le rapport physiologique et pathologique. Cette analogie des effets produits par l'inflammation sur les séreuses est affirmé encore dans de nombreux passages de Hunter. Par exemple:

Lorsque l'inflammation prend naissance dans des parties qui sont plus ou moins transparentes, leur transparence est diminuée. C'est ce qu'on voit surtout dans les membranes comme celles qui tapissent des cavités ou qui recouvrent des organes contenus dans ces cavités; telle est la pie-mère... ${ }^{33}$

L'idée que les maladies locales n'attaquent pas toujours les organes en totalité, mais parfois leurs tissus constituants séparément a été développée tout à fait explicitement par John Hunter dès le début de la seconde moitié du XVIII ${ }^{\mathrm{e}}$ siècle. Dans l'œuvre du médecin écossais, on trouve, en effet, de nombreux passages où cette idée est affirmée clairement.

Voici l'un des textes les plus explicites, où l'on voit fonctionner le concept de maladie tissulaire:

Conformément à ce qui a été dit ci-dessus, les membranes enveloppantes ne sont pas unies par l'espèce de sympathie en question avec les parties qu'elles enveloppent ou qu'elles tapissent, et les parties qui recouvrent les membranes enveloppantes ou qui sont tapissées par elles ne sympathisent point non plus avec elles dans la période adhésive de l'inflammation. Ainsi, le péritoine est à la fois une membrane qui tapisse et une membrane qui enveloppe; il en est de même pour la plèvre; si le péritoine qui tapisse la cavité abdominale s'enflamme, son inflammation n'affecte point les parois de cette cavité; et si le péritoine qui recouvre un des viscères s'enflamme, il n'affecte point ce viscère. Le péritoine peut être enflammé dans sa totalité, comme dans la fièvre puerpérale, sans que les parois de l'abdomen et les membranes propres des intestins en soient affectées. D'un autre côté, si les parois de l'abdomen ou les membranes propres des intestins sont enflammées le péritoine n'est point affecté. Le même principe conduit à la ligne de démarcation qui existe entre l'inflammation des poumons et celle de la plèvre. Mais je présume qu'il y a des rapports sympathiques plus intimes entre les cellules aériennes des poumons et le tissu réticulaire ou tissu unissant qui réunit ces celulles entre elles, 
et réciproquement, qu'il n'en existe entre les parties ci-dessus mentionnées; et cela peut dépendre du peu d'épaisseur des parois des cellules aériennes. C'est aussi d'après le même principe que l'inflammation de la pie-mère se continue rarement dans la substance du cerveau, quoique la pie-mère puisse être considérée en partie comme une continuation des mêmes vaisseaux.

La continuité des parties ne favorise point l'extension de l'inflammation. Ainsi quand un intestin est enflammé, l'inflammation ne se communique point au péritoine qui tapisse l'abdomen, quoi qu'il soit en contact avec lui ${ }^{44}$.

Dans ce seul passage, nous avons plusieurs exemples de cas où la maladie n'atteint pas l'organe en sa totalité, mais seulement certains de ses éléments constituants. Ainsi, une méningite due à l'inflammation de la pie-mère n'est pas une inflammation de tout le cerveau. De même, la pleurésie n'est pas une inflammation de tout le poumon. De même encore, la péritonite n'est pas une inflammation de toute la région abdominale et elle peut ne pas affecter les membranes muqueuses des intestins.

Hunter établit dans ce texte (comme dans celui que nous avons cité juste avant) un principe fondamental de la pathologie tissulaire: dans un même système d'organes, lorsque certains des tissus constituants sont atteints par la maladie, les autres tissus peuvent demeurer sains. Par exemple, dans la région de l'abdomen, lorsque les membranes appartenant au système séreux sont enflammées, les membranes muqueuses peuvent n'être pas atteintes par l'inflammation et, inversement, lorsque c'est les membranes muqueuses qui sont en état d'inflammation dans les intestins, les membranes séreuses demeurent intactes ${ }^{45}$.

Bichat ne s'inspire-t-il pas sur ce point directement de Hunter quand il écrit:

L'affection d'un organe n'est point une conséquence nécessaire de celle de sa membrane séreuse, et réciproquement, souvent l'organe s'affecte sans que la membrane devienne malade. Par exemple, dans l'opération de l'hydrocèle, le testicule reste presque constamment intact au milieu de l'inflammation de sa tunique vaginale: l'inflammation de la membrane muqueuse des intestins n'est point une suite de celle de leur enveloppe péritonéale; et réciproquement dans les diverses affections catarrhales aïgues des organes à membrane muqueuses au-dedans et séreuses au-dehors, on ne voit point celle-ci s'enflammer. En un mot, les affections des membranes muqueuses sont partout très distinctes de celles des séreuses, quoique communément toutes deux concourent à la formation d'un même organe. Il est évident qu'une ligne de démarcation si réelle dans les affections en suppose inévitablement une dans l'organisation: la vie des membranes séreuses est donc entièrement isolée de celle de leurs organes correspondants ${ }^{46}$.

La similitude de ce texte avec différents passages de Hunter (dont ceux que nous venons de citer $)^{47}$ est vraiment frappante. E. H. Ackerknecht attribue à Bichat l'idée qu'il y a indépendance des membranes enveloppantes par rapport aux 
organes enveloppés (et réciproquement) dans l'inflammation et qu'il y a une vie propre aux différents tissus ${ }^{48}$. En réalité, Bichat reprend cette problématique de Hunter, mais en lui donnant une forme peut-être un peu trop dogmatique: pour le médecin écossais, en effet, l'inflammation pouvait aussi se propager de la membrane à l'organe (il en donne certains exemples dont celui de l'inflammation de la tunique vaginale qui peut s'étendre au testicule ${ }^{49}$ ) ou affecter deux tissus différents simultanément. Par exemple, selon Hunter, le poumon connaît des modes d'inflammation distincts selon le tissu qui est affecté:

Les poumons sont le siège d'un double mode d'action dans leur état inflammatoire; parce que, dans leur structure, ils participent du caractère des deux ordres de partie, savoir, les cavités circonscrites et les surfaces de sécrétion. Le tissu cellulaire des poumons admet aussi facilement l'inflammation adhésive que le tissu cellulaire de toutes les autres parties du corps; mais la surface intérieure des cellules aériennes, de même que la membrane muqueuse de l'urètre, passe directement à l'inflammation suppurative, et la matière qui est ainsi formée doit être rejetée par la toux ${ }^{50}$.

Cependant, il ne faut pas conclure à partir de là que l'inflammation ne peut exister que dans l'un des systèmes tissulaires à l'exclusion de l'autre. Hunter pense différemment, puisqu'il ajoute:

Or, l'inflammation de l'un de ces tissus ne peut guère exister sans que l'autre soit également enflammé, et c'est peut-être pour cela que l'inflammation de ces viscères est traitée avec si peu de succès ${ }^{51}$.

On peut donc se demander si Bichat n'effectue pas une transcription quelque peu unilatérale de la problèmatique de Hunter quand il écrit:

Prenons l'exemple du poumon. Cet organe se compose de la plèvre, du tissu pulmonaire et de la membrane interne. Dans la pleurésie, il n'y a que la plèvre d'enflammée, le tissu pulmonaire et la membrane muqueuse sont intacts. Dans la péripneumonie, c'est au contraire le poumon proprement dit, tandis que les deux membranes sont saines. De même les toux catarrhales sont exclusives à la membrane muqueuse, tandis que le parenchyme et la membrane séreuse sont dans l'état naturel. Cet exemple peut servir de terme de comparaison pour tous les autres organes ${ }^{52}$.

S'il est vrai - et Hunter l'a reconnu - que l'inflammation prend ordinairement naissance sur un seul tissu et que «dans un grand nombre de maladies, on voit les parties se conserver distinctes les unes des autres aussi longtemps qu'elles le peuvent $»,{ }^{53}$ ce qui explique qu'il existe «une ligne de démarcation entre l'inflammation des poumons et celle de la plèvre», ${ }^{54}$ il n'en demeure pas moins que l'inflammation peut gagner les tissus distincts de celui où elle est d'abord apparue et que par la suite, elle peut exister simultanément dans les tissus différents 
(même si elle existe avec des caractères différents dans ces différents tissus). Bichat affirme que les toux catarrhales sont exclusives à la membrane muqueuse tandis que le parenchyme et la membrane séreuse sont sains. Hunter était loin d'ignorer que dans les catarrhes, c'est la membrane muqueuse qui est atteinte et qui est le siège de la suppuration ${ }^{55}$. Cependant, pour l'auteur écossais, quand la membrane muqueuse du poumon est enflammée, il y a assez souvent sympathie entre ce tissu et le tissu cellulaire (l'inverse est vrai aussi). C'est pourquoi, dans le passage que nous avons déjà cité ${ }^{56}$, Hunter, après avoir affirmé que ses principes conduisent à établir une ligne de démarcation entre l'inflammation des poumons et celle de la plèvre, fait l'observation suivante:

Mais je présume qu'il y a des rapports sympathiques plus intimes entre les cellules aériennes des poumons et le tissu réticulaire ou tissu unissant qui réunit ces cellules entre elles, et réciproquement, qu'il n'en existe entre les parties ci-dessous mentionnées; et cela peut dépendre du peu d'épaisseur des parois des cellules aériennes ${ }^{57}$.

En 1819, l'auteur de l'article «Membrane» du Dictionnaire des Sciences médicales attribue à Pinel et à Bichat l'idée que dans l'inflammation d'un organe composé de plusieurs tissus, alors que l'un de ces tissus est altéré, les autres peuvent conserver leur état normal:

La classification des membranes a fourni beaucoup d'idées nouvelles aux médecins; un viscère est formé par la réunion de plusieurs de ces tissus: on considérait jadis son inflammation comme une maladie toujours la même, mais depuis la distinction des membranes en genres, les genres de phlegmasies ont été multipliés. Un intestin est formé principalement d'une tunique péritonéale, d'une tunique musculaire et d'une tunique interne ou muqueuse; les nosologistes ont pensé que l'un de ces tissus pouvait être enflammé isolément; que l'inflammation de chacun d'eux avait des caractères particuliers, et que dans une phlegmasie du conduit intestinal plus ou moins étendue, il pouvait y avoir tantôt seulement une péritonite, tantôt seulement une inflammation de la membrane muqueuse ${ }^{58}$.

En fait, dans le premier quart du $\mathrm{XIX}^{\mathrm{e}}$ siècle, les cliniciens de l'école de Pinel et de Bichat n'avaient rien découvert sur ce plan qui ne fût déjà chez Hunter environ un demi-siècle plutôt. Il est d'ailleurs très significatif à cet égard, que ce soit justement l'exemple de l'inflammation de l'intestin, sur lequel Hunter avait insisté à plusieurs reprises, qui permette encore en 1819 d'illustrer le principe selon lequel la maladie peut ne pas affecter l'organe en son entier, mais seulement certains des tissus qui le composent.

En fonction de ce qui précède, on peut affirmer que John Hunter est sans doute un des auteurs auxquels Bichat fait référence lorsqu'il parle de plusieurs «méde- 
cins célèbres» qui ont établi une ligne de démarcation entre les différents tissus de l'économie, à partir de l'observation des caractères variés que prend l'inflammation sur les différentes membranes ${ }^{59}$.

Il reste à se demander maintenant quels sont les autres médecins célèbres auxquels Bichat fait référence ${ }^{60}$. Nous pensons qu'il peut s'agir de cliniciens comme les «Viennois» G. Van Swieten (1700-1772), A.de Haen (1704-1776), M. Stoll (1742-1788); les Italiens G. B. Morgagni (1682-1771) (nul autre que lui!) et D. Cotugno (1736-1822); les Anglais Robert Whytt (1714-1766), W.Cullen (1710-1790), W. Hunter (1718-1783), A. Monro primus (1697-1767), et A. Monro secundus (1733-1817), W. Hewson (1739-1774), W.Stark (1740-1770), W. Cruikshank (1745-1800); le Français Th. de Bordeu (1722-1776), etc. Nous analyserons dans une prochaine étude ${ }^{61}$ les contributions respectives de ces différents auteurs à la pathologie tissulaire.

On peut souligner cependant dès à présent, que vu le nombre et l'importance des médecins qui ont fourni des résultats dans ce domaine dans la seconde moitié du XVIII ${ }^{\mathrm{e}}$ siècle, il n'est plus possible d'affirmer que l'articulation de l'anatomie pathologique et de la clinique ne pouvait se produire que dans les hôpitaux universitaires parisiens à partir de la Révolution ${ }^{62}$. Cette interprétation n'est même pas adéquate pour la France, puisque la mise en rapport de l'anatomie pathologique et de la clinique s'y était déjà effectuée à partir de la seconde moitié du XVIII ${ }^{\mathrm{e}}$ siècle dans l'école pratique de dissection de Paris (là aussi à partir de la problématique chirurgicale) et dans les hôpitaux civils et militaires ${ }^{63}$.

Dans un travail antérieur ${ }^{64}$, nous avions montré comment Bichat, à travers Pinel, s'était inspiré de la problématique d'un auteur anglais: J. C. Smyth. Nous avions cherché aussi à prouver que ce qui avait rendu possible l'œuvre de Bichat, c'était l'articulation qu'il avait opérée entre les thèses de Smyth (à travers Pinel) et celles de John Hunter (ainsi que celles des autres élèves de l'école de W. Hunter). La présente étude renforce encore, nous semble-t-il, la thèse que nous avions avancée.

Notes

${ }^{1}$ P.Triaire, Récamier et ses contemporains. 1774-1852. Etudes d'histoire de la médecine au $X V I I I^{e}$ et au XIX ${ }^{e}$ siècles. Paris, 1899, p.112.

${ }^{2}$ Xavier Bichat, Traité des membranes. Edition de F. Magendie. Paris, 1827, p. 4.

${ }^{3}$ Ibid., p. 3.

${ }^{4}$ Ibid.

${ }^{5}$ Ibid., pp. 3-4. 
${ }^{6}$ Ibid., p. 12.

${ }^{7}$ Georges Cuvier, Histoire des sciences naturelles depuis leur origine jusqu'à nos jours... Paris (1841-45), vol.5, p.58.

${ }^{8}$ Othmar Keel, La généalogie de l'histopathologie. Une révision déchirante. (Vrin) Paris, 1979.

${ }^{9}$ Bichat, op. cit., p. 3 .

${ }^{10}$ Bichat a nécessairement eu connaissance des travaux de J.Hunter par l'enseignement de son maître, le chirurgien Desault, qui faisait très souvent référence au chirurgien écossais dans ses cours. Cf. X. Bichat (ed.), Euvres chirurgicales de Desault, 3 vols., Paris, 1798-1799.

${ }^{11}$ Cf. Keel, op. cit.

${ }^{12}$ Cf. EEuvres complètes de John Hunter. Edition de James Palmer. Trad. fr. G. Richelot, 4. vols., Paris, 1843.

${ }^{13}$ Cf. Keel, op. cit., chap. IV, note 46.

${ }^{14}$ Op. cit., vol.1, p.418.

15 Ibid.

16 Ibid.

17 Ibid.

18 Ibid.

19 Ibid., p. 421.

${ }^{20}$ Ibid., pp.507-508. Palmer écrit à ce sujet: «Hunter fait observer que le tissu vasculaire résiste plus que tous les autres aux effets de l'ulcération et de la gangrène.» Cf. Hunter, op. cit., vol.3, p.588.

${ }^{21}$ Ibid., p. 562.

${ }^{22}$ Ibid.

${ }^{23}$ Ibid., p. 565.

${ }^{24}$ Ibid., p. 577.

${ }^{25}$ Ibid.

26 Ibid.

${ }^{27}$ Ibid., p. 577.

28 Ibid.

${ }^{29}$ Ibid., p. 639.

30 Ibid.

31 Ibid.

${ }^{32}$ Ibid., p. 477.

33 Ibid., p. 480.

${ }^{34}$ Ibid., p. 466.

35 Alard, Alibert, Barbier et al. (eds.), 60 vols., Paris, 1812-1822.

${ }^{36}$ Ibid., vol. 32 (1819), p. 222.

${ }^{37}$ Cf. Hunter, op. cit., vol. 3.

${ }^{38}$ Cf. Hunter, Ibid., p. 13, et aussi Keel, op. cit., chap.III.

${ }^{39}$ Op. cit., vol.3, p. 326.

${ }^{40}$ Op. cit., vol.1, p.XLIII.

${ }^{11} \mathrm{Op}$. cit., vol.1, p.497. Cf. chez Hunter l'analyse très fouillée des altérations anatomopathologiques du système séreux dans l'inflammation. $O p$. cit., vol.3, p.391sq. 
42 Ibid.

43 Ibid., vol. 3, p. 363.

${ }^{44}$ Ibid., pp.329-330.

45 Cf. note 41.

46 Traité..., pp.101-102.

${ }^{47}$ Cf. note 41 et note 44 .

${ }^{48}$ Erwin H.Ackerknecht, Medicine at the Paris Hospital (1794-1848), Baltimore, 1967, p.53.

${ }^{49}$ Cf. note 41.

${ }^{50}$ Hunter, op. cit., vol.1, p.451.

${ }^{51}$ Ibid. Ailleurs, Hunter développe encore plus explicitement ces mêmes idées. Cf. op. cit., vol. 3, p. 447 .

${ }^{52}$ Xavier Bichat, Cours d'anatomie pathologique, Paris, 1825, p.12. La position de Bichat est plus nuancée sur ces questions dans l'Anatomie générale: elle correspond d'avantage donc à celle de Hunter: "Quand la membrane des bronches est le siège d'une catarrhe, la plèvre ne s'en ressent que peu, et réciproquement dans la pleurésie la première ne s'affecte presque pas. Dans la péripneumonie, lorsqu'une énorme infiltration annonce sur le cadavre l'excès d'inflammation qui a lieu pendant la vie dans le tissu pulmonaire, ses deux surfaces séreuses et muqueuses ne paraissent souvent pas avoir été affectées.» (Vol.1, p. LXXXVI.) C'est nous qui mettons en italique.

Bichat ajoute encore un peu plus loin: «N'exagérons pas cependant cette indépendance où les tissus d'un organe sont les uns des autres sous le rapport des maladies: la pratique nous démentirait. Nous verrons le système cellulaire être souvent une voie de communication, non seulement d'un tissu à l'autre dans le même organe, mais encore d'un organe à son voisin. Aussi dans beaucoup de maladies chroniques, toutes les parties du même organe s'altèrent peu à peu, et à l'ouverture du cadavre la totalité de cet organe, nous paraît affectée, quoiqu'un seul de ces tissus l'ait été primitivement.

Voyez la phtisie développant dans le principe quelques petits tubercules dans le tissu pulmonaire, envahissant souvent à la fin, la plèvre, la membrane bronchique, etc.

... un tissu étant d'abord affecté dans un organe, communique peu à peu son affection aux autres, et ... ce serait mal juger du siège primitif que de l'estimer par les parties où il a lieu à l'instant où on examine le sujet.» (Vol.1, p. XCI.)

${ }^{53}$ Op. cit., vol.3, p. 329.

${ }^{54}$ Ibid., p. 330.

${ }^{55}$ Ibid., vol.1, p. 464 .

${ }^{56}$ Cf. note 44.

${ }^{57}$ Op. cit., vol.3, p. 329.

${ }^{58}$ Op. cit., vol.32, p. 210 .

${ }^{59}$ L'existence d'une pathologie tissulaire chez John Hunter n'a pas été reconnue par les historiens de la médecine, même dans le cas où ils se sont occupés exclusivement de cet auteur (cf. par ex. Jessie Dobson, John Hunter, Edimbourg et Londres, 1969).

Le seul auteur moderne qui, à notre connaissance, ait signalé l'intérêt de Hunter pour la pathologie des tissus est E.H. Ackerknecht: "In his ideas of local inflammation of tissues and sympathies, Broussais was immediately preceded by John Hunter, Bichat, Marandel and Pinel" (op. cit. note 48, p. 70). P. Klemperer signale l'importance de Hunter 
pour une conception dynamique du processus anatomo-pathologique mais il continue à attribuer à Bichat le concept de maladie tissulaire: "But the works of his pupils, foremost among them Matthew Baillie, William Stark, and John Abernethy, illustrate the guiding principle of his thoughts about pathologic anatomy: i.e., to search for the evolution of the morbid lesion. In other words, he was concerned with the morbid process and not merely with the static organ alteration." "The pathology of Morgagni and Virchow", J. of the Mount Sinaï Hospital, 1957, 23: p.32. Klemperer ajoute un peu plus loin: "The ideas of Bichat who, in the early years of the century, had tried to establish the elementary composition and functional properties of the animal body by careful analysis of the constituent tissues... etc." (p.32). Nous avons montré ailleurs que l'importance de Hunter pour la pathologie tissulaire avait été reconnue par certains auteurs du XIX ${ }^{\mathrm{e}}$ siècle (Broussais, J.F.Palmer, etc.). Cf. Keel, op. cit.

${ }^{60}$ On pourrait citer de très nombreuses références de Bichat aux travaux de différents auteurs sur les membranes. Par exemple: «Quelles seraient les fonctions des villosités de la membrane pituitaire, de la tunique interne de l'urètre, de la vessie, etc. si elles n'ont pas rapport à la sensibilité de ces membranes? Les expériences microscopiques de Liberkuhn, sur l'ampoule des villosités intestinales, ont été contredites par celles de Hunter, de Cruikshank et surtout de Hewsson». Anatomie générale (Edition de 1836: Encyclopédie des Sciences Médicales), p.395. Autre référence directe à Hunter: p.394.

${ }^{61}$ Sur ce point, quelques indications in Keel, op. cit.

${ }^{62}$ Sur cette question ef. Othmar Keel, Cabanis et la généalogie épistémologique de la médecine clinique, Thèse Ph.D. Univ. McGill, 1977.

${ }^{63}$ Cf. Marie-José Imbault-Huart, L'Ecole pratique de dissection de Paris de 1750 à 1822, (Thèse) Paris, 1973.

${ }^{64}$ O. Keel, op. cit. (1979). Cf. note 8.

\section{Summary}

According to a widely held opinion in the history of medicine, it is only at the end of the eighteenth century that the concepts of tissue pathology were introduced into medicine through the works of Philippe Pinel and Xavier Bichat. However, the present study advances evidence in support of view that the basic concepts of tissue pathology had already been functioning in John Hunter's medico-surgical problematic for approximately half a century before the appearance of the first books by Pinel and Bichat.

\section{Othmar Keel}

Institut d'Histoire et de Sociopolitique des Sciences

Université de Montréal

Case postale 6128, Succursale «A»

Montréal, P.Q.; Canada H3C 3J7 